\title{
Safety of Discharge of Seniors from the Emergency Department to the Community
}

Jane McCusker, Danièle Roberge, Alain Vadeboncoeur and Josée Verdon

\begin{abstract}
This study investigated the safety of discharge of seniors (aged 65 and over) from Quebec emergency departments (EDs) to the community. Data from a 2006 survey of key informants at 103 Quebec adult non-psychiatric EDs were linked to data on a sample of 172,927 seniors who were discharged home from one of the EDs during the period February 2004-January 2005. During the 30 days after the ED visit, $1.0 \%$ of patients died, $5.0 \%$ returned to the ED and were admitted to hospital, $16.0 \%$ returned to the ED but were not admitted and $29.2 \%$ were prescribed a potentially inappropriate medication. Larger, urban EDs treated a higher-risk patient population (older, greater co-morbidity), and these seniors had worse outcomes. A minority of EDs, regardless of their size and the characteristics of patients treated, systematically provided services to improve the safety of discharge. Resources and services need to be improved in EDs, particularly those that serve higher-risk populations (e.g., systematic approaches to the identification and management of high-risk seniors, with appropriate referrals to community services), in the hospital (e.g., increased accessibility to acute care beds) and in the community (e.g., increased accessibility to home care, outpatient geriatric assessment and primary medical care).
\end{abstract}

\section{Background}

Although there have been numerous studies of patient safety in the hospital setting, little research has focused on the emergency department (ED). The ED, a critical interface between the hospital and community, presents major challenges to patient safety that are of particular importance in the rapidly growing population of seniors (defined here as those aged 65 and over). Higher levels of co-morbidity, physical and cognitive impairment and polypharmacy make seniors particularly vulnerable to adverse outcomes after an ED visit, such as functional decline, mortality and early return ED visits (McCusker et al. 1999). In addition, the busy ED environment promotes a focus on rapid treatment and disposition of patients. A failure to detect problems that require investigation or intervention, such as delirium or medical conditions that may have contributed to a fall, may result in discharge without appropriate referral for assessment and lead to increased rates of return visits and other adverse outcomes (Hohl et al. 2001; Kakuma et al. 2003; McCusker et al. 1999, 2000a, 2000b). A failure to review patient medications may increase adverse effects resulting from polypharmacy and inappropriate prescribing (Beers et al. 1990; Hohl et al. 2001). Limited access to patient information from the primary physician, home care services, previous hospitalization or previous ED visits, for example, may lead to inappropriate treatment and disposition (Stiell et al. 2003). Finally, a failure of ED staff to transmit information about the visit to the 
primary physician and to community services (e.g., home care) may result in delays before the patient is eventually reassessed, treated or provided services (McCusker et al. 2001).

In light of these challenges, safer discharge of seniors from the ED may involve improvements in services and staff to assess the patient needs, detect geriatric problems, review and modify medications, access community services and transfer appropriate information to family physicians. Thus, this study aimed to describe (1) the services provided to seniors in different types of EDs, (2) the profiles of seniors who are discharged home from different types of EDs and (3) the outcomes of these seniors during the 30 days after an $\mathrm{ED}$ visit. (hospital discharge [MedEcho], physician billing and medication prescription [Régie de l'assurance maladie du Québec; RAMQ] databases) for the Quebec population were used to describe patient characteristics, index ED visits and outcomes. ED visits were identified in the RAMQ database using a validated method (Dendukuri et al. 2005). The index ED visit was defined as the first visit during the 12-month study period of February 2004 to January 2005 . The study sample was composed of 172,927 non-institutionalized patients aged 65 or over who had an index ED visit during the 12-month period to one of 103 Quebec adult, non-psychiatric EDs and were discharged home. Figure 1 illustrates how the sample was derived.

\section{Methods}

\section{Study Design and Sample}

This study linked data from ED registries, physician billings, hospital discharges and a survey of key informants. The study protocol was approved by the Commission d'accès à l'information and the Research Ethics Committee of St. Mary's Hospital.

\section{ED Registry Data}

Provincial ED registry data were used to compute the volume of visits, the number of ED beds (stretchers), the daily average length of stay on ED beds and a measure of crowding - the average daily ratio of patients to ED beds. Because these data cannot be linked at the patient level to other administrative databases, we identified individual patient ED visits from the administrative databases described below.

\section{Provincial \\ Administrative \\ Databases}

Three linked provincial administrative databases

\section{Figure 1. Study flowchart}

RAMQ users age $65+$ with at least 1 bill in the RAM0 database between April 1, 2003, and March 31, 2005, and at least 1 ED claim between Feb. 1, 2004, and Jan. 31, 2005 $(n=301,232)$

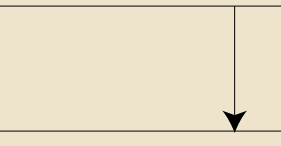

Index visit Feb. 1, 2004-Jan. 31, 2005 $(n=298,047)$

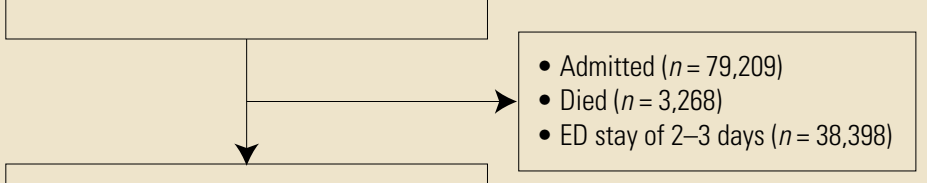

Alive and non-hospitalized at index visit $(n=177,172)$

- Long-term care resident $(n=3,598)$

- No hospital assigned ( $n=530)$

- Mental health hospital ( $n=117$ patients at 2 hospitals)

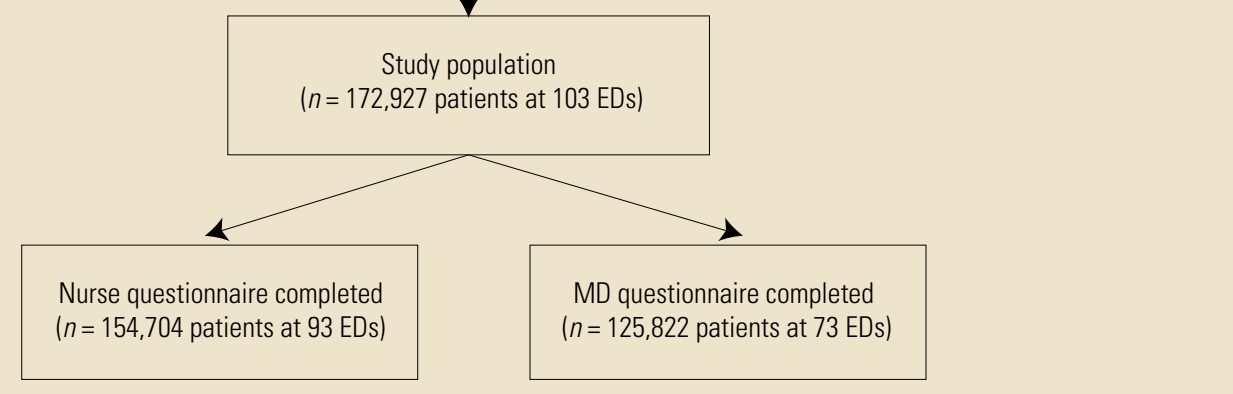

ED = emergency department; $\mathrm{MD}$ = physician; RAMQ: Régie de l'assurance maladie du Québec (medication prescription database). 
Patient characteristics included age, sex, co-morbidity (D'Hoore et al. 1996) and use of healthcare services during the previous 12 months (hospital days, physician office visits). A 30-day time frame was used for the measurement of outcomes: death, emergency hospital admission, return ED visit without hospitalization, visit to the primary physician (McCusker et al. 2003b) and the prescription of a potentially inappropriate medication (based on the modified Beers criteria, excluding estrogens [Fick et al. 2003]).

\section{Survey of Key Informants}

A survey was conducted during the summer of 2006 using questionnaires directed to the ED chief physician and head nurse. If there was an individual responsible for the care of seniors, this person assisted the head nurse with the relevant sections of the questionnaire. Response rates were $71 \%$ for physicians and $90 \%$ for nurses. Head nurses were queried on the following: nurse staffing per shift, non-medical professionals involved in ED care of seniors, ED geriatrics services (the use of standardized screening and assessment tools, a discharge planning protocol, post-ED telephone follow-up) and satisfaction with home care services. Chief physicians were queried on medical ED staffing (number of physicians per weekday shift), the availability of geriatrics and psychogeriatrics consultation, whether there is a pharmacist or pharmacy technician in the $\mathrm{ED}$, computerization and the transfer of information to and from family physicians.

\section{ED Classification}

Other data on ED characteristics (location, university affiliation, number of beds and level of care classified as primary, secondary and tertiary based on comprehensiveness of resources) were obtained from the Quebec Ministry of Health and Social Services. An ED classification was developed based on the following correlated ED characteristics: location, university affiliation, number of beds and level of care. (Further details on the methodology used to develop this classification are available from the authors.) Three ED types were identified: (1) large EDs, mostly with 21 or more ED beds and located mainly in the Montreal area $(n=30)$; (2) medium-size EDs, mostly with 14-20 ED beds and located mainly in urban areas outside Montreal $(n=29)$; and (3) small EDs, often based in health centres rather than hospitals, most with fewer than 14 ED beds and located in rural areas $(n=44)$ (Table 1$)$.

\section{Results}

Table 1 shows selected characteristics of the three ED types, including the variables used to create the classification. The mean length of stay for patients on ED beds was 24 hours or more among one in five EDs, with no significant difference by ED size. There were significantly higher rates of crowding at larger EDs.

\section{Services Provided by Different Types of ED}

In general, the staff, resources and clinical tools of interest were available in only a minority of EDs (Table 2); there were, nevertheless, important differences by ED type. The ratio of physicians to ED beds was lower at larger EDs. Some resources and tools were significantly more available at larger EDs, such as on-site pharmacy staff, a team leader for geriatric care, geriatrics consultation, systematic screening and administrative and clinical software. Community linkages with the family doctor and home care services also differed by ED size - in smaller EDs, there were higher rates of satisfaction with home care and of the transmission of information from the ED to the family doctor.

\section{Patient Profiles by ED Type}

Patient characteristics indicated a population with high rates of hospitalization and physician visits in the previous year (mean of 2.5 days and 9.2 visits, respectively). There were significant differences by ED type in many patient characteristics, most of which showed a gradient across the type of ED (Table 3). (It should be noted that even small clinical differences in patient variables may be statistically significant because of the large sample size.) Seniors discharged home from larger EDs were older, were more often female, had higher co-morbidity scores and had higher rates of hospitalization and physician visits in the previous year.

\section{Patient Outcomes}

Table 4 shows the frequency of 30-day outcomes and measures of the process of care among seniors discharged home from the three types of ED. The most serious outcomes - death (1.0\%), and/or return to the ED with hospital admission (5.0\%), were found in $5.6 \%$ of the sample (some patients experienced both outcomes). A further $16.0 \%$ made an ED return visit but were not admitted. Approximately three of 10 seniors filled a prescription for a potentially inappropriate medication, and the same proportion visited their primary physician.

Even after controlling statistically for the patient characteristics shown in Table 3, there were significant differences in outcomes among the three types of ED (data not shown): seniors who made an index visit at a large or medium ED were significantly more likely than those visiting a small ED to die or be admitted to hospital; in contrast, they were less likely to make a return ED visit without hospitalization. ED type was not related to visits to the primary physician or to the prescription of a potentially inappropriate medication.

\section{Discussion}

This study represents a first step toward the assessment of the safety of discharge of seniors from the ED, an important topic that has received relatively little attention in the research literature. It should be noted that the study was conducted in Quebec, 
Table 1. Characteristics of three types of ED

\begin{tabular}{|c|c|c|c|c|c|}
\hline \multirow[b]{2}{*}{ Variable } & \multirow[b]{2}{*}{$\begin{array}{c}\text { Total } \\
(N=103) \\
\%\end{array}$} & \multicolumn{3}{|c|}{ Type } & \multirow[b]{2}{*}{$p$ Value* } \\
\hline & & $\begin{array}{c}\text { Large } \\
\begin{array}{c}(n=30) \\
\%\end{array}\end{array}$ & $\begin{array}{c}\text { Medium } \\
(n=29) \\
\%\end{array}$ & $\begin{array}{c}\text { Small } \\
(n=44) \\
\%\end{array}$ & \\
\hline \multicolumn{6}{|l|}{ Variables comprising ED classification } \\
\hline \multicolumn{6}{|l|}{ Level of care } \\
\hline Tertiary or specialized & 20 & 60 & 7 & 0 & $<.0001$ \\
\hline Secondary & 36 & 40 & 86 & 2 & \\
\hline Primary & 24 & 0 & 7 & 50 & \\
\hline Not classified & 21 & 0 & 0 & 48 & \\
\hline University affiliated & 39 & 73 & 52 & 7 & $<.0001$ \\
\hline No. of ED beds & & & & & $<.0001$ \\
\hline $1-13$ & 49 & 0 & 38 & 95 & \\
\hline $14-20$ & 21 & 13 & 52 & 5 & \\
\hline $21-56$ & 30 & 87 & 10 & 0 & \\
\hline Geographic location & & & & & $<.0001$ \\
\hline Montreal/Laval & 17 & 53 & 7 & 0 & \\
\hline Other urban & 50 & 47 & 93 & 23 & \\
\hline Rural & 33 & 0 & 0 & 77 & \\
\hline \multicolumn{6}{|l|}{ Other variables } \\
\hline Mean ED length of stay (h) for patients on beds ${ }^{\dagger}$ & & & & & .89 \\
\hline $0-11$ & 59 & 51 & 59 & 68 & \\
\hline $12-23$ & 22 & 24 & 21 & 20 & \\
\hline $24-47$ & 14 & 18 & 15 & 9 & \\
\hline $48+$ & 5 & 7 & 6 & 3 & \\
\hline \multicolumn{6}{|l|}{ Crowding (average daily patient-to-ED bed ratio) ${ }^{\dagger}$} \\
\hline $\operatorname{Low}(<0.80)$ & 24 & 3 & 17 & 54 & $<.0001$ \\
\hline Medium (0.80-1.33) & 51 & 60 & 48 & 43 & \\
\hline $\operatorname{High}(\geq 1.34)$ & 25 & 37 & 34 & 4 & \\
\hline
\end{tabular}

$E D=$ emergency department.

'Missing data for 16-20 EDs, mostly in the small category.

${ }^{*}$ Chi-square test. 
Table 2. Staff and services by ED type

\begin{tabular}{|c|c|c|c|c|c|c|}
\hline \multirow[b]{2}{*}{ Variable } & \multirow[b]{2}{*}{$n^{*}$} & \multirow[b]{2}{*}{$\begin{array}{c}\text { Total } \\
(N=103)\end{array}$} & \multicolumn{3}{|c|}{ Type } & \multirow[b]{2}{*}{ p Value } \\
\hline & & & $\begin{array}{l}\text { Large } \\
(n=30)\end{array}$ & $\begin{array}{c}\text { Medium } \\
(n=29)\end{array}$ & $\begin{array}{c}\text { Small } \\
(n=44)\end{array}$ & \\
\hline \multicolumn{7}{|l|}{ Staffing } \\
\hline Ratio of nurses on weekday shift to no. of ED beds (mean) & 93 & 0.5 & 0.5 & 0.5 & 0.6 & .10 \\
\hline Ratio of MDs on weekday shift to no. of ED beds (mean) & 73 & 0.2 & 0.1 & 0.2 & 0.3 & .007 \\
\hline Pharmacist in ED & 73 & $32 \%$ & $70 \%$ & $17 \%$ & $17 \%$ & $<.0001$ \\
\hline \multicolumn{7}{|l|}{ Geriatrics resources } \\
\hline Staff involved in care of seniors & 93 & & & & & \\
\hline Liaison nurse & & $44 \%$ & $58 \%$ & $46 \%$ & $34 \%$ & .16 \\
\hline Geriatrics clinical nurse & & $9 \%$ & $15 \%$ & $8 \%$ & $5 \%$ & .32 \\
\hline Social worker & & $42 \%$ & $50 \%$ & $46 \%$ & $34 \%$ & .39 \\
\hline Team leader for geriatric care & 93 & $25 \%$ & $54 \%$ & $20 \%$ & $10 \%$ & $<.001$ \\
\hline Geriatrics or psychogeriatrics consultation available & 73 & $34 \%$ & $60 \%$ & $22 \%$ & $27 \%$ & .02 \\
\hline \multicolumn{7}{|l|}{ Clinical tools } \\
\hline Systematic screening of seniors & 93 & $30 \%$ & $46 \%$ & $39 \%$ & $15 \%$ & .02 \\
\hline Standardized assessment tools & 93 & & & & & \\
\hline Function & & $16 \%$ & $27 \%$ & $15 \%$ & $10 \%$ & .21 \\
\hline Cognition & & $17 \%$ & $31 \%$ & $15 \%$ & $10 \%$ & .11 \\
\hline Other & & $14 \%$ & $27 \%$ & $8 \%$ & $10 \%$ & .10 \\
\hline Discharge planning protocol & 93 & $13 \%$ & $12 \%$ & $19 \%$ & $10 \%$ & .54 \\
\hline Post-ED follow-up: sometimes/often & 93 & $28 \%$ & $48 \%$ & $20 \%$ & $21 \%$ & .06 \\
\hline Computerization & 73 & & & & & \\
\hline Administrative & & $43 \%$ & $80 \%$ & $44 \%$ & $17 \%$ & $<.0001$ \\
\hline Clinical & & $53 \%$ & $90 \%$ & $57 \%$ & $27 \%$ & $<.0001$ \\
\hline Pharmaceutical & & $14 \%$ & $20 \%$ & $13 \%$ & $10 \%$ & 63 \\
\hline \multicolumn{7}{|l|}{ Community linkages } \\
\hline Satisfaction with home care services (mean, range 1-5) & 93 & 3.6 & 3.1 & 3.6 & 3.9 & .0004 \\
\hline Information usually sent to family doctor & 73 & $43 \%$ & $40 \%$ & $17 \%$ & $63 \%$ & .004 \\
\hline Information usually received from family doctor & 73 & $19 \%$ & $5 \%$ & $22 \%$ & $27 \%$ & 14 \\
\hline
\end{tabular}

$\mathrm{ED}=$ emergency department; $\mathrm{MD}=$ physician .

*Data were collected from 93 head nurses at 26 large, 26 medium and 41 small EDs; and from 73 ED chief physicians at 20 large, 23 medium and 30 small EDs. 
Table 3. Characteristics of patients aged 65 and over by ED type

\begin{tabular}{|c|c|c|c|c|c|}
\hline \multirow[b]{2}{*}{ Variables } & \multirow[b]{2}{*}{$\begin{array}{c}\text { Total } \\
(N=172,927)\end{array}$} & \multicolumn{3}{|c|}{ Type } & \multirow[b]{2}{*}{$p$ Value } \\
\hline & & $\begin{array}{c}\text { Large } \\
(n=79,880)\end{array}$ & $\begin{array}{l}\text { Medium } \\
(n=49,540)\end{array}$ & $\begin{array}{c}\text { Small } \\
(n=43,507)\end{array}$ & \\
\hline Age (yr, mean) & 76.4 & 76.7 & 76.4 & 75.9 & $<.0001$ \\
\hline Female $(\%)$ & 58.3 & 59.5 & 58.0 & 56.5 & .0004 \\
\hline Charlson Comorbidity Index (mean)* & 1.3 & 1.4 & 1.3 & 1.1 & $<.0001$ \\
\hline Hospital days in previous year (mean) & 2.5 & 2.7 & 2.6 & 1.9 & $<.0001$ \\
\hline Physician visits in previous year (mean) & 9.2 & 10.8 & 8.7 & 7.0 & $<.0001$ \\
\hline
\end{tabular}

$\mathrm{ED}=$ emergency department.

*Higher score indicates more serious chronic conditions.

Table 4. Patient 30-day outcomes and process of care by ED type

\begin{tabular}{|l|c|c|c|c|}
\hline & & \multicolumn{3}{|c|}{ Type } \\
\cline { 3 - 5 } Outcome & $\begin{array}{c}\text { Total } \\
(N=172,927) \\
\%\end{array}$ & $\begin{array}{c}\text { Large } \\
(n=79,880) \\
\%\end{array}$ & $\begin{array}{c}\text { Medium } \\
(\boldsymbol{n}=\mathbf{4 9 , 5 4 0 )} \\
\%\end{array}$ & $\begin{array}{c}\text { Small } \\
(\boldsymbol{n}=\mathbf{4 3 , 5 0 7 )} \\
\%\end{array}$ \\
\hline Death & 1.0 & 1.2 & 1.2 & 0.6 \\
\hline Return to ED with hospital admission & 5.0 & 5.5 & 5.0 \\
\hline Return to ED without hospital admission & 16.0 & 14.5 & 15.0 & 20.0 \\
\hline Potentially inappropriate medication prescription & 29.2 & 29.2 & 30.1 & 28.0 \\
\hline Primary physician visit & 32.0 & 33.4 & 31.7 & 29.8 \\
\hline
\end{tabular}

$\mathrm{ED}=$ emergency department.

where ED stays of over 24 hours or even several days are not unusual, particularly in the Montreal area. The study provides a portrait of different types of Quebec EDs - large, medium and small - and shows great variation in both the staffing and services provided and in the seniors who were discharged home from different types of ED.

The study results highlight some potential safety concerns for seniors discharged to the community. Although the largest EDs treated the oldest seniors with higher rates of co-morbidity, hospitalization and doctor visits, their average length of stay for seniors on beds was similar to that in smaller EDs. This suggests a greater difficulty in accessing in-patient beds in the largest EDs, with resulting higher rates of crowding (McCusker et al. 2007), which has been linked to reduced quality of care, increased rates of return ED visits and mortality (McCusker et al. 2007; Richardson 2006; Sprivulis et al. 2006). Other safety concerns in larger EDs are the lower physician staffing ratios and the perceived lack of availability of home care services.

Overall, only a minority of EDs offered the types of services that can improve the safety of discharge of seniors to the community, such as systematic patient assessment and discharge planning (Hastings and Heflin 2005). Even among large EDs serving higher-risk seniors, only about half did any systematic geriatric screening, about one third used standardized tools to assess patient function or cognition, $12 \%$ had a discharge planning protocol and $40 \%$ usually sent patient information to the family doctor. A standardized assessment of seniors while in the ED, along with the transfer of key information to 
the family physician and links to community services, is costeffective because it both reduces further functional decline and increases continuity of care (McCusker et al. 2003a, 2003b). The implementation of such approaches needs to be prioritized in the context of a rapidly aging population.

Seniors discharged home from larger EDs tended to be somewhat more severely ill and to include a higher proportion of high-risk or frail elderly patients; these individuals likely required substantially more medical and support services in the community. Rates of death (1.0\%) and total return ED visits with and without hospital admission (21.0\%) during the 30 days after the index visit were within the range of those previously reported (Caplan et al. 2004; Lowenstein et al. 1986; McCusker et al. 2007; Mion et al. 2003; Ross et al. 2003); however, these outcomes differed by type of ED. Seniors discharged from large or medium EDs had higher rates of more severe 30-day outcomes (death or hospitalization), which was consistent with their poor clinical status. In contrast, return visits without hospital admission were more frequent in small, rural EDs. There are several possible reasons for this occurrence. Rural physicians often practise in multiple settings, including the ED, and may conduct patient follow-up in the ED (Haggerty et al. 2007). Return ED visits may reflect inadequate treatment at the initial visit. In addition, a small proportion may be planned visits to follow-up on treatment, especially in smaller EDs where other locations for follow-up may be less available.

Only $32 \%$ of patients visited their primary physician during the 30 days after being released home from an ED. This proportion appears low and may reflect the use of the ED as a substitute for primary care, a lack of accessibility to a primary physician or a lack of communication between the ED and the primary physician (McCusker et al. 2003a). An ED visit is a sentinel event for seniors, and a routine follow-up visit to a family physician may prevent a return ED visit (Caplan et al. 2004).

\section{Limitations}

Several limitations of this study should be noted. First, the aims of the study were descriptive; further research needs to examine the links between patient characteristics, services and outcomes. Second, data on patient characteristics and outcomes were derived from administrative databases, which contain limited data of uncertain validity (e.g., diagnostic data). Third, ED registry data have important limitations: they are not currently linked at the patient level to other administrative databases, and data on length of stay and crowding were not available for some smaller EDs that either have few beds or do not report these data consistently. Fourth, the survey data on the availability of specific services at each ED did not indicate which of these services were actually used or whether they were used for high-risk patients. Fifth, it was not possible to distinguish between planned and unplanned return ED visits. Sixth, it was not possible to determine which medication prescriptions were given to patients at the ED visit because the location of the prescribing physician was not available. Further research could focus on changes in prescriptions from before to after the ED visit to identify which medications were discontinued and which were initiated. Finally, these data from Quebec may not be generalizable to other provinces or countries.

\section{Conclusion and Recommendations}

The results of this study confirm that seniors are a high-risk population whose needs may not be adequately addressed by existing ED and community services (McCusker et al. 1999). While some of the safety concerns identified in this study may require a redeployment of governmental resources to those EDs, hospitals and communities with higher-risk patients and the most severe ED crowding, other concerns can be addressed within the ED by reorganizing existing resources and implementing protocols (e.g., for the systematic identification of seniors at risk and appropriate discharge planning). Below we present improvements that can be done in the ED itself, the hospital and the community.

\section{Improvements in the Emergency Department}

In the $\mathrm{ED}$, a basic requirement is appropriate staffing. Ideally, all EDs should have access to geriatrics nurses, teams or consultants. In small EDs where it is not feasible to have dedicated geriatrics staff, existing staff could be trained in geriatrics, and referral networks could be developed. Evidence-based services that could be implemented by a liaison nurse or social worker include standardized, systematic ED-based screening, assessment and discharge planning, with referrals as needed for community services (Hastings and Heflin 2005; Verdon and McCusker 2005; Warburton et al. 2004). High-risk seniors need more complex discharge planning. A standardized approach, using protocols, should be followed in all EDs. An increased involvement of pharmacists in the ED or the use of software designed to identify inappropriate medications may help to reduce medication-related adverse events and return ED visits (Bizovi et al. 2002; Forster et al. 2004; Langdorf et al. 2000). The length of ED stays should be limited - longer stays may be particularly detrimental for seniors. EDs should monitor their return visits to determine whether they may have been prevented with better management at the initial visit. Most importantly, regional health planners need to recognize the differences between different types of EDs. The case-mix of EDs should be measured, taking into account not only age but level of illness and functional status; in this manner, resources could be matched to the needs of the patients.

\section{Improvements in the Hospital}

In the hospital, rapid accessibility to acute care beds is needed, 
particularly in those larger EDs that suffer from high rates of crowding. An increased availability of acute care beds or innovative solutions for the use of these beds in large urban hospitals (e.g., establishing a short stay unit for seniors) may help to accommodate the increased need and reduce the adverse outcomes associated with overcrowding (Forster et al. 2003). Channels of transfer from the ED to subacute care, rehabilitation or convalescent centres could be developed for appropriate patients.

\section{Improvements in the Community}

At-risk seniors require follow-up in the community. It must therefore be a priority to ensure that they have access to essential community services (e.g., home care, geriatric assessment, primary medical care) and experience continuity of care (Ionescu-Ittu et al. 2007). This is of particular concern in Quebec where the availability of family physicians is worse than that in other Canadian provinces (Canadian Institute of Health Information 2005; Sanmartin 2006). Programs are needed to allow rapid home assessment and routine follow-up visits to the family doctor. This will require standardized communication between the EDs and the community, appropriate funding of these resources and an adequate supply of family doctors to take on new patients. Improvements in the organization of community-based care for seniors with chronic illnesses and the development of a continuum of care between primary and secondary levels of care may also help to reduce ED visits (Castonguay et al. 2008; Clair 2000). Such improvements in access to services and continuity of care will augment patient safety in this highrisk population. $\mathrm{HQ}$

\section{Acknowledgements}

We acknowledge the contributions of the following team members: Antonia Arnaert, Bruce Brown, Monica Cepoiu, Antonio Ciampi, Danielle Larouche, Louise Mallet, Lise Poissant and Jacques Brunet.

This study was funded by the Canadian Patient Safety Institute.

\section{References}

Beers, M.H., M. Storrie and G. Lee. 1990. "Potential Adverse Drug Interactions in the Emergency Room." Annals of Internal Medicine 112: $61-64$.

Bizovi, K.E., B.E. Beckley, M.C. McDade, A.L. Adams, R.A. Lowe, A.D. Zechnich and J.R. Hedges. 2002. "The Effect of ComputerAssisted Prescription Writing on Emergency Department Prescription Errors." Academic Emergency Medicine 9(11): 1168-75.

Canadian Institute of Health Information. 2005. Healthcare in Canada. Ottawa, ON: Author.

Caplan, G.A., A.J. Williams, B. Daly and K. Abraham. 2004. “A Randomized Controlled Trial of Comprehensive Geriatric Assessment and Multidisciplinary Intervention after Discharge of Elderly from the Emergency Department - The DEED II Study." Journal of the American Geriatrics Society 52(9): 1417-23.
Castonguay, C., J. Marcotte and M. Venne. 2008. En Avoir Pour Notre Argent - Rapport du Groupe de Travail sur le Financement du Systeme de Santé. Québec, PQ: Gouvernement du Québec.

Clair, M. 2000. Les Solutions Émergentes: Rapport et Recommandations. Québec, PQ: Gouvernement du Québec.

Dendukuri, N., J. McCusker, F. Bellavance, S. Cardin, J. Verdon, I. Karp and E. Belzile. 2005. "Comparing the Validity of Different Sources of Information on Emergency Department Visits: A Latent Class Analysis." Medical Care 43(3): 266-75.

D'Hoore, W., A. Bouckaert and C. Tilquin. 1996. "Practical Considerations on the Use of the Charlson Comorbidity Index with Administrative Data Bases." Journal of Clinical Epidemiology 49(12): 1429-33.

Fick, D.M., J.W. Cooper, W.E. Wade, J.L. Waller, R.J. Maclean and M.H. Beers. 2003. "Updating the Beers Criteria for Potentially Inappropriate Medication Use in Older Adults." Archives of Internal Medicine 163(22): 2716-24.

Forster, A.J., H.D. Clark, A. Menard, N. Dupuis, R. Chernish, N. Chandok, A. Khan and C. van Walraven. 2004. "Adverse Events among Medical Patients after Discharge from Hospital." Canadian Medical Association Journal 170(3): 345-49.

Forster, A.J., I. Stiell, G. Wells, A.J. Lee and C. van Walraven. 2003. "The Effect of Hospital Occupancy on Emergency Department Length of Stay and Patient Disposition." Academic Emergency Medicine 10(2): 127-33.

Haggerty, J.L., D. Roberge, R. Pineault, D. Larouche and N. Touati. 2007. "Features of Primary Healthcare Clinics Associated with Patients' Utilization of Emergency Rooms: Urban-Rural Differences. Healthcare Policy 3(2): 72-85.

Hastings, S.N. and M.T. Heflin. 2005. "A Systematic Review of Interventions to Improve Outcomes for Elders Discharged from the Emergency Department." Academic Emergency Medicine 12(10): 978-86.

Hohl, C.M., J. Dankoff, A. Colacone and M. Afilalo. 2001. "Polypharmacy, Adverse Drug-Related Events, and Potential Adverse Drug Interactions in Elderly Patients Presenting to an Emergency Department." Annals of Emergency Medicine 38(6): 666-71.

Ionescu-Ittu, R., J. McCusker, A. Ciampi, A. Vadeboncoeur, D. Roberge, D. Larouche, J. Verdon and R. Pineault. 2007. "Continuity of Primary Care and Emergency Department Utilization among Elderly People." Canadian Medical Association Journal 177(11): 1362-68.

Kakuma, R., G. Galbaud du Fort, L. Arsenault, A. Perrault, R.W. Platt, J. Monette, Y. Moride and C. Wolfson. 2003. "Delirium in Older Emergency Department Patients Discharged Home: Effect on Survival." Journal of the American Geriatrics Society 51(4): 443-50.

Langdorf, M.I., J.C. Fox, R.S. Marwah, B.J. Montague and M.M. Hart. 2000. "Physician versus Computer Knowledge of Potential Drug Interactions in the Emergency Department." Academic Emergency Medicine 7(11): 1321-29.

Lowenstein, S.R., C.A. Crescenzi, D.C. Kern and K. Steel. 1986. "Care of the Elderly in the Emergency Department." Annals of Emergency Medicine 15(5): 528-35.

McCusker, J., F. Bellavance, S. Cardin, E. Belzile and J. Verdon. 2000a. "Prediction of Hospital Utilization among Elderly Patients during the Six Months after an Emergency Department Visit." Annals of Emergency Medicine 36(5): 438-45.

McCusker, J., F. Bellavance, S. Cardin, S. Trépanier, J. Verdon and O. Ardman. 1999. "Detection of Older People at Increased Risk of Adverse Health Outcomes after an Emergency Visit: The ISAR 
Screening Tool." Journal of the American Geriatrics Society 47(10): 1229-37.

McCusker, J., N. Dendukuri, P. Tousignant, J. Verdon, L. Poulin De Courval and E. Belzile. 2003a. "Rapid Two-Stage Emergency Department Intervention for Seniors: Impact on Continuity of Care." Academic Emergency Medicine 10(3): 233-43.

McCusker, J., O. Ardman, F. Bellavance, E. Belzile, S. Cardin and J. Verdon. 2001. "Use of Community Services by Seniors before and after an Emergency Visit." Canadian Journal on Aging 20(2): 193-209.

McCusker, J., P. Jacobs, N. Dendukuri, E. Latimer, P. Tousignant and J. Verdon. 2003b. "Cost-Effectiveness of a Brief 2-Stage Emergency Department Intervention for High Risk Elders: Results of a QuasiRandomized Controlled Trial." Annals of Emergency Medicine 41(1): 45-56.

McCusker, J., R. Ionescu-Ittu, A. Ciampi, A. Vadeboncoeur, D. Roberge, D. Larouche, J. Verdon and R. Pineault. 2007. "Hospital Characteristics and Emergency Department Care of Older Patients Are Associated with Return Visits." Academic Emergency Medicine 14(5): 426-33.

McCusker, J., S. Cardin, F. Bellavance and É. Belzile. 2000b. "Return to the Emergency Department among Elders: Patterns and Predictors." Academic Emergency Medicine 7(3): 249-59.

Mion, L.C., R.M. Palmer, S.W. Meldon, D.M. Bass, M.E. Singer, S.M. Payne, L.J. Lewicki, B.L. Drew, J.T. Connor, J.W. Campbell and C. Emerman. 2003. "Case Finding and Referral Model for Emergency Department Elders: A Randomized Clinical Trial." Annals of Emergency Medicine 41(1): 57-68.

Richardson, D.B. 2006. "Increase in Patient Mortality at 10 Days Associated with Emergency Department Overcrowding." Medical Journal of Australia 184(5): 213-16.

Ross, M.A., S. Compton, D. Richardson, R. Jones, T. Nittis and A. Wilson. 2003. "The Use and Effectiveness of an Emergency Department Observation Unit for Elderly Patients." Annals of Emergency Medicine 41(5): 668-77.

Sanmartin, C. 2006. "Experiencing Difficulties Accessing FirstContact Health Services in Canada." Healthcare Policy 1(2): 103-19.

Sprivulis, P.C., J.-A. Da Silva, I.G. Jacobs, A.R. Frazer and G.A. Jelinek. 2006. "The Association between Hospital Overcrowding and Mortality among Patients Admitted via Western Australian Emergency Departments." Medical Journal of Australia 184(5): 208-12.

Stiell, A., A.J. Forster, I.G. Stiell and C. van Walraven. 2003. "Prevalence of Information Gaps in the Emergency Department and the Effect on Patient Outcomes." Canadian Medical Association Journal 169(10): 1023-28.

Verdon, J. and J. McCusker. 2005. “Intervention Rapide en Deux Étapes, a L'Urgence, pour les Patients Âgés: Impact sur la Continuité de Soins." Info Urgence Automne: 33.

Warburton, R.N., B. Parke, W. Church and J. McCusker. 2004. "Identification of Seniors at Risk: Process Evaluation of a Screening and Referral Program for Patients Aged 75 and Over in a Community Hospital Emergency Department." International Journal of Health Care Quality Assurance 17(6): 339-48.

\section{About the Authors}

Jane McCusker, MD, DrPH, heads the Department of Clinical Epidemiology and Community Studies at St. Mary's Hospital in Montreal and is Professor in the Department of Epidemiology, Biostatistics and Occupational Health at McGill University.
Danièle Roberge, $\mathrm{PhD}$, is an adjunct professor at the Université de Sherbrooke and her research is focused on the organization of emergency rooms, primary care and cancer care.

Alain Vadeboncoeur, MD, is a specialist in emergency medicine and Chief of the Medical Emergency Service, Montreal Heart Institute, member of the Montreal Heart Institute Research Centre and also a clinical associate professor, medical emergency division, department of family and emergency medicine, Université de Montreal.

Josée Verdon, MD, is a specialist in internal medicine and geriatrics at McGill University Health Centre, Royal Victoria Hospital and also an assistant professor, McGill University, Department of Medicine, Division of Geriatrics. Her areas of interest are based on care of seniors in the emergency room, interdisciplinary team approach, and models and process of care.

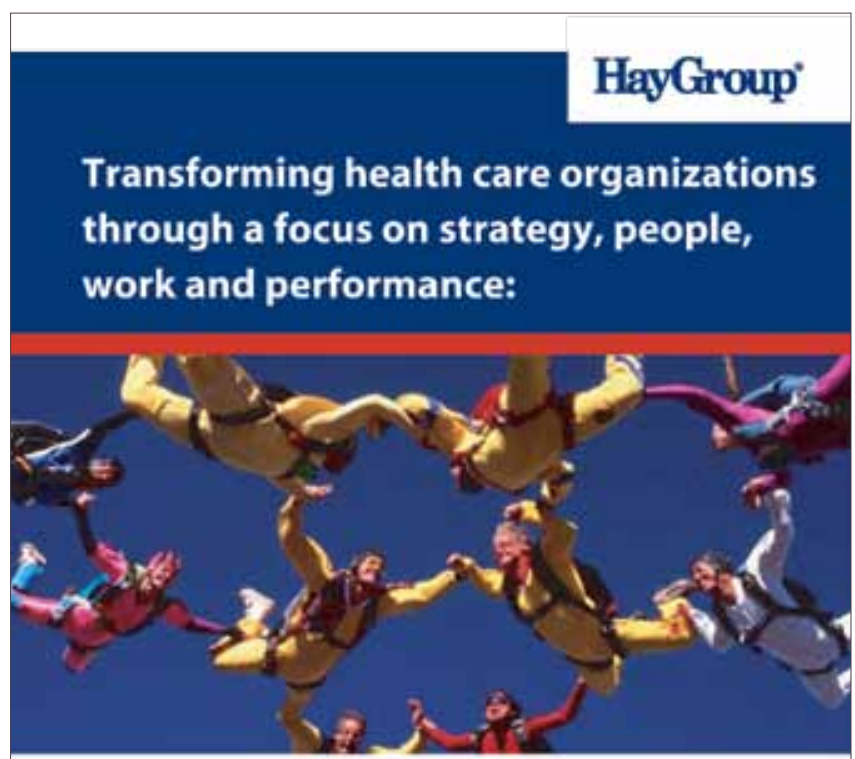

- Planning

- Operational Improvement

- Organizational Effectiveness

- Human Resources Management

- Reward Programs

Contact:

Mark Hundert, National Director

Tel: (416) 868-1371 Fax: (416) 868-0362 